Please send trade news information and illustrations to Arveen Bajaj at the BDJ, 64 Wimpole Street, London W1G 8YS. Trade news is supplied as a service to the reader and does not imply endorsement by the BDJ. Normal and prudent research should be exercised before purchase of use of any product mentioned.

\section{TRADE NEWS \\ WHAT'S NEW}

\section{Complete brushing}

New to The Dental Directory is the Oral-B Sonic Complete. It has a three speed brushing action (clean, soft and massage) and a cushioned gum bumper on the brush head to act as a buffer for teeth and gums against the 31,000 vibrations per minute.

It also incorporates a two-minute timer that signals every 30 seconds to encourage quadrant-by-quadrant brushing as well as a slim, lightweight handle.

To compliment the range of electric toothbrushes sold, the company also stocks a selection of replacement brush heads. Reader response number 50

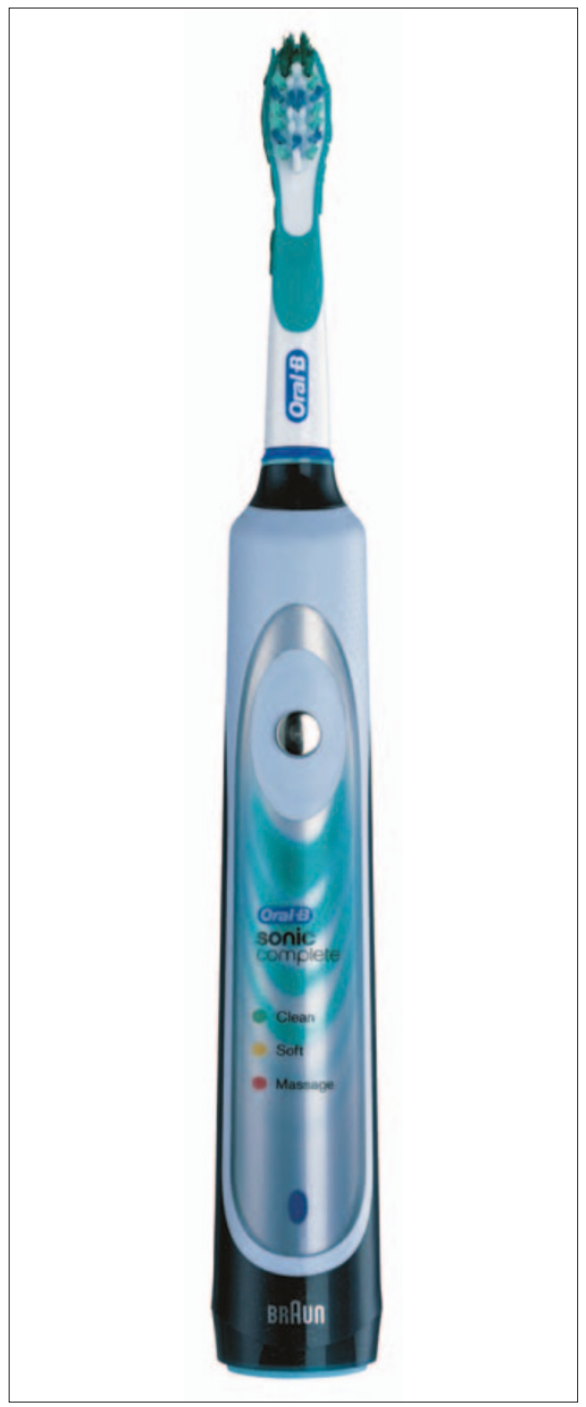

\title{
No shrinkage
}

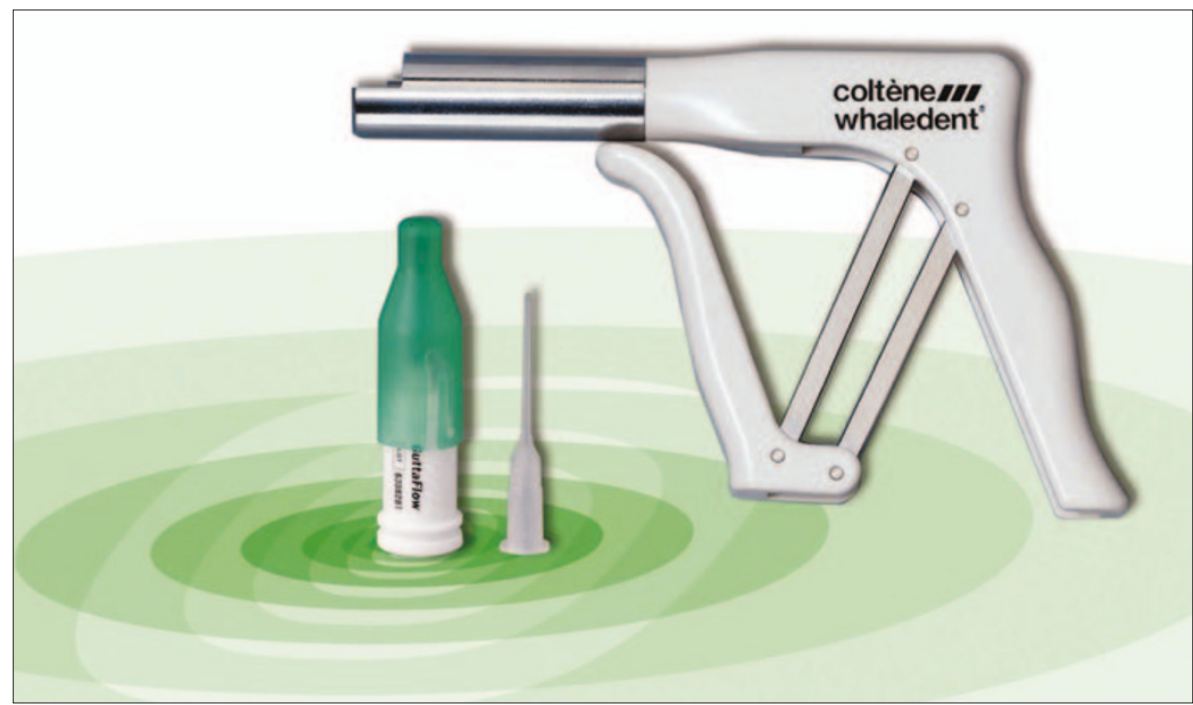

GuttaFlow from Coltene/Whaledent is a cold filling system for the obturation of root canals which combines gutta-percha and sealer in one product. Its material properties consist of insolubility, no shrinkage, and good biocompatibility. An application system has also been developed which enables a simple, safe and hygienic operation.

According to the company, GuttaFlow is the first non-heated free-flow gutta-percha

\section{Tapered bristles}

Manx Healthcare have extended the Endekay range and launched a new generation toothbrush into the UK market.

The Endekay Microfine toothbrush has tapered bristles and two levels of bristle heights.

Its makers claim that the regular and tapered bristles make the ideal configuration to make cleaning as efficient as possible, both on the tooth surface and within the gum margin and interdental areas.

The bristle ends bend back at the slightest pressure, reducing the likelihood of harming the gums.

The Endekay Microfine toothbrush comes in two adult sizes and one child's size.

Reader response number 52 that does not shrink. It guarantees reliable dosage and mixing through the 'disposable' capsule system. The material's flow properties allow optimum distribution in the root canal and can be removed easily during retreatment.

One introductory kit contains 20 capsules of GuttaFlow, 20 canal tips, one dispenser and two packs of gutta-percha points.

Reader response number 51

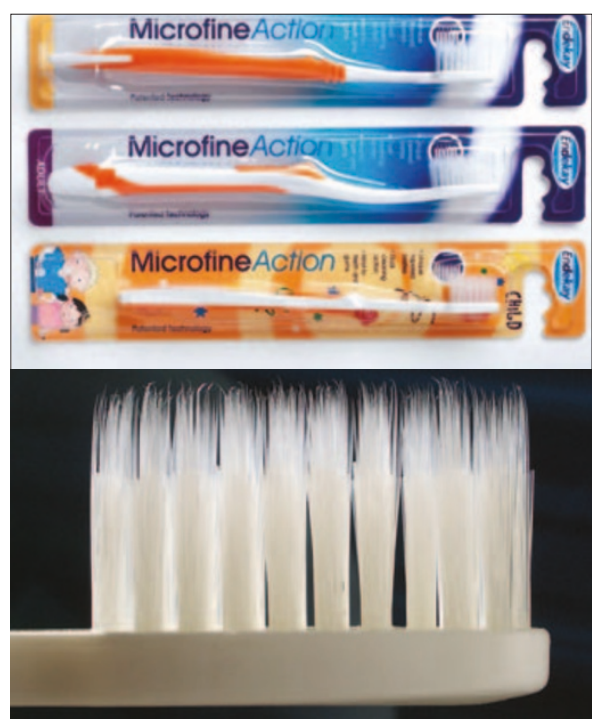




\section{IMPRESSION MATERIALS \&t ORAL HEALTH FINANCE}

\section{Spreading the cost}

With the 3dee patient loan service, patients can spread the cost over a period that suits their financial circumstances.

According to Frank Taylor and Associates, 3dee Treatment Funding encourages patient acceptance of higher value treatment plans.

The product enables patients to spread the cost of dental treatment up to $£ 15,000$ (or more by special arrangement), over an agreed period of up to five years.

The company offers competitive interest rates, an interest free option or buy now pay later, and provides comprehensive practice support and staff training.

Reader response number 53

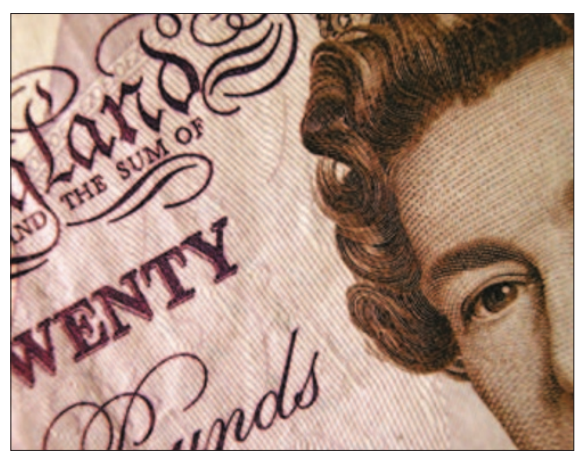

\section{Tropical mix}

DB Orthodontics Limited stock a wide range of tropical mixing bowls and spatulas which are available in a range of colours. Used in combination with the tropical spatulas, they are flexible enough to make blending alginates into a smooth bubble free mix even easier, the company claims. They are manufactured from a flexible, translucent material and the latest addition is an autoclavable blue plastic spatula.

Reader response number 54

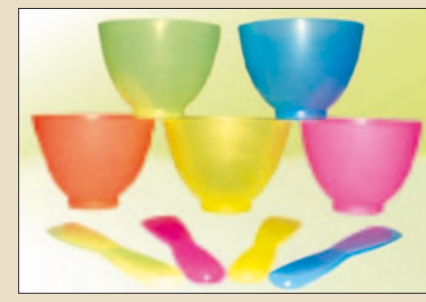

\section{Bite registration}

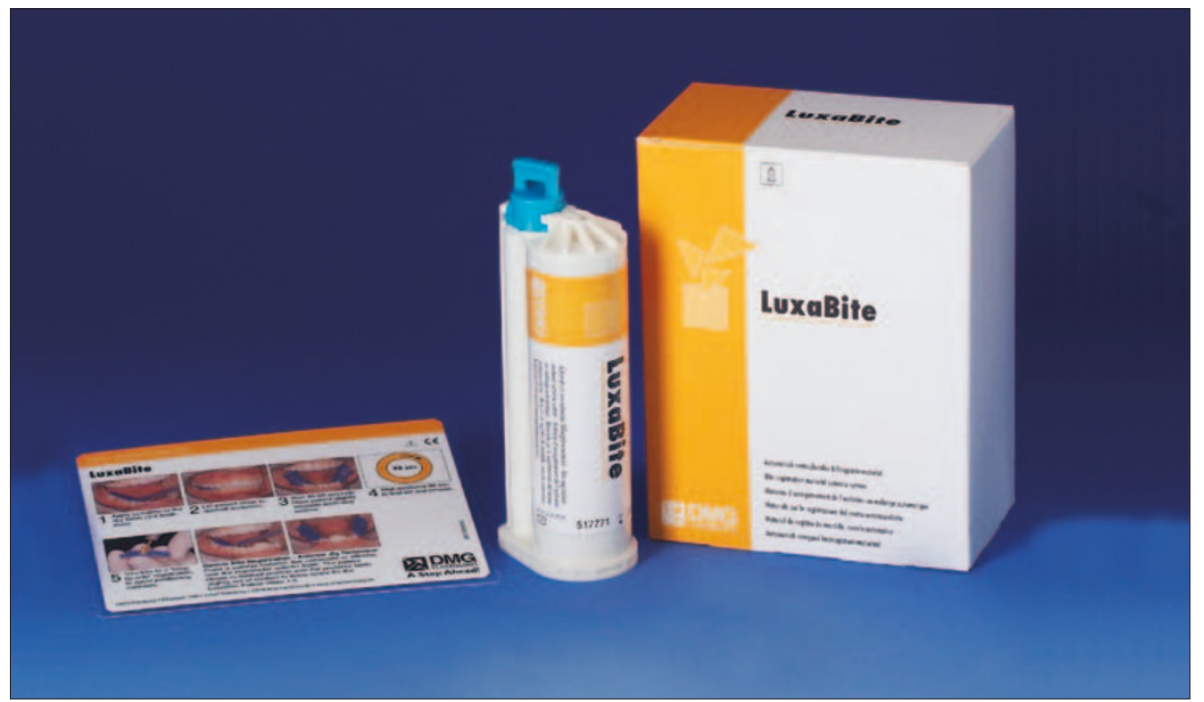

New LuxaBite Automix from DMG is a cartridge dispensed, rigid bite registration material, which delivers accurate and reliable occlusal records, according to the company. It combines superior handling properties with a firm set to yield the most accurate and stable bite registration records possible and does not distort, which can reduce the risk of costly remakes.

LuxaBite's thixotropy ensures minimal resistance to closure and consequently less risk of inaccuracy. While its high final hardness means it exhibits no compression

or flexing when mounting the models, its hardness also makes it break resistant, yet easy to trim. Odourless and taste free it has a contrasting blue colour, which is easy to visualise intraorally and on the model.

Automatically mixed and dispensed, this eliminates the risk of under-dosing or over-dosing and ensures delivery of a consistent quality of homogeneous and bubble-free material directly onto the occlusal surface. This can ensure optimum physical and chemical properties, minimal waste and maximum efficiency, DMG claims.

Reader response number 55

\section{Faster impressions}

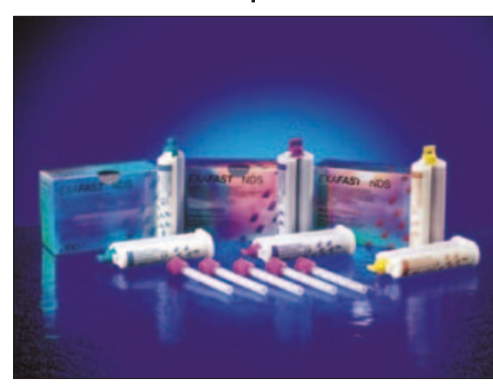

GC has produced a range of impression materials that set in super fast time with Exafast NDS. The Exafast NDS family of addition cured silicones allows the clinician to produce very smooth and precise impressions in two minutes. GC Exafast Putty is an extremely fast setting putty material, for use in the single step technique.

In combination with other GC Exafast NDS materials it has a working time of 45 seconds and setting time of two minutes 15 seconds. The Exafast wash material is available in three viscosities and all are supplied in cartridges for speed and ease of application. As the material sets quickly there is less risk of distortion due to movement during the settings and its makers claim it has a high degree of elasticity, tear resistance and good dimensional stability.

Reader response number 56 


\section{New finance product launched}

A new finance product, enabling dentists to offer patients interest-free loans of up to $£ 25,000$ for private dental treatment has been launched this month.

Denplan Enhance enables more patients to afford high value dentistry such as implants, cosmetic or orthodontic treatment by spreading the cost over monthly instalments, Denplan claims.

Subject to credit-checks, loans can be offered to all patients, whether Denplan, NHS or private, and treatment can be booked in immediately.

An on-the-spot patient application process allows immediate confirmation of loans from $£ 500$ to $£ 25,000$, repayable by direct debit over six to 36 months (depending on the loan term). There are no set-up costs for dentists and patients are not obliged to pay a deposit.

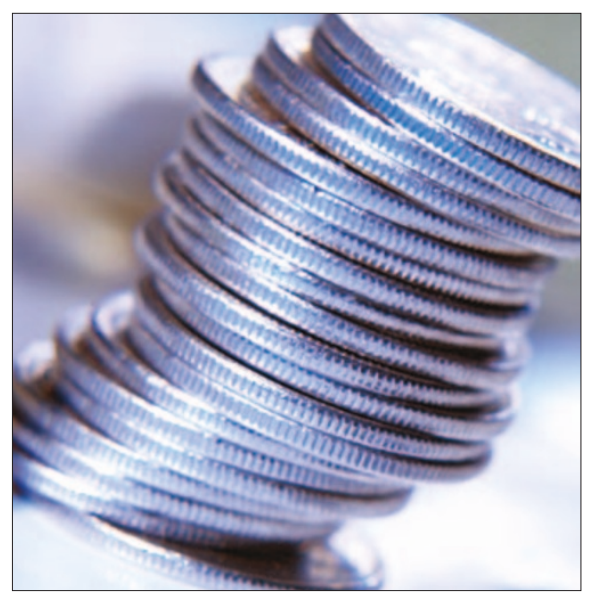

Dentists receive their payment up-front and loans are secured without financial risk to the practice.

Reader response number 57

\section{New mixing machine}

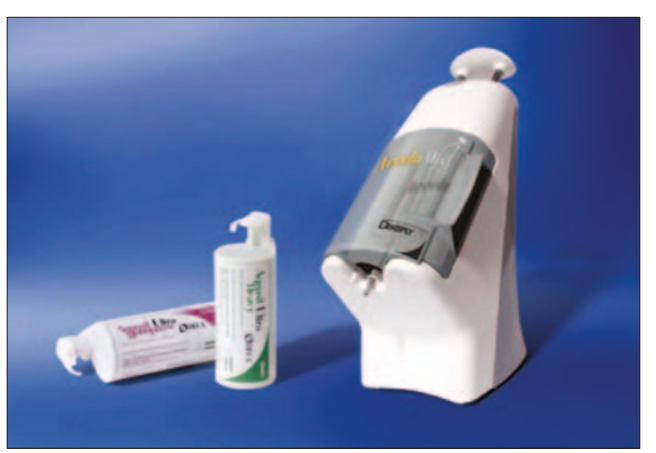

Dentsply UK has introduced two new products to its Aquasil Ultra product range. The new mixing machine, Accelamix and the Decatm 380 Hard Cartridges ensure the production of consistent, quality Aquasil Ultra, the company claims.

It features a one-step, one-motion plunger set-up, with no knobs to turn, faster loading, a special reverse speed which reduces dripping, a safety shutdown that reduces the risk of foil packs bursting and a compact design with fast and reliable performance. The Decatm 380 Hard Cartridges also have additional benefits including a one-component system that eliminates the need for a delivery cylinder and less material waste as depleted cartridges have less than $2 \mathrm{ml}$ of material remaining inside, ensuring that they are cost-effective.

Reader response number 58

\section{Low-splatter formula}

The Nupro prophy paste range from Dentsply has been extended with the introduction of mint flavour in economysized jars with fluoride.

The jar range now offers practices the benefits of Nupro's low-splatter formula in two flavours - orange and mint - to increase patient choice.

Benefits include leaving a refreshing taste in the mouth and mint may be preferred by adults over orange. They are available in jars with fluoride and in unit dose cups, without fluoride, while orange Nupro gives a zesty finish to prophylaxis.

The product is also available in fine grit for paediatric prophylaxis and in jars or unit dose cups, without fluoride.

In addition the special low-splatter formula means less cleaning up, which can make for an easier working environment. Reader response number 59

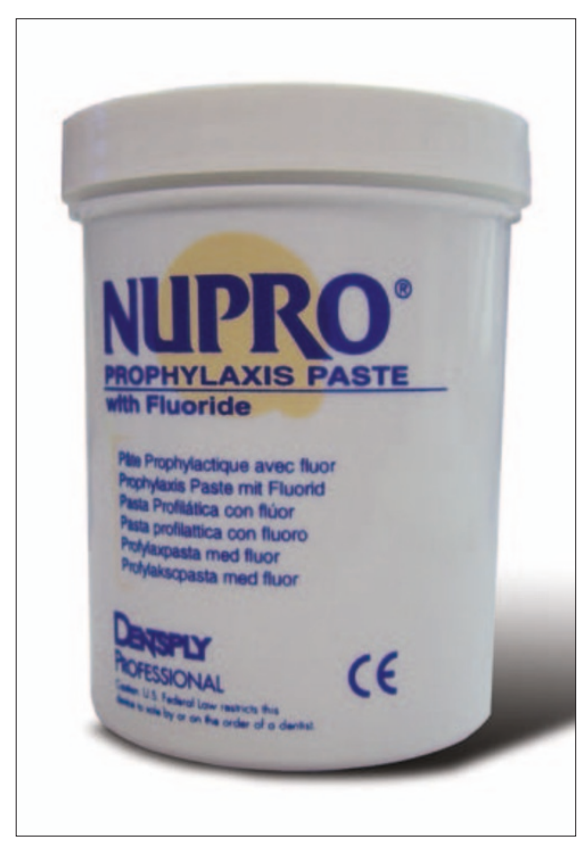




\section{Flexible impressions}

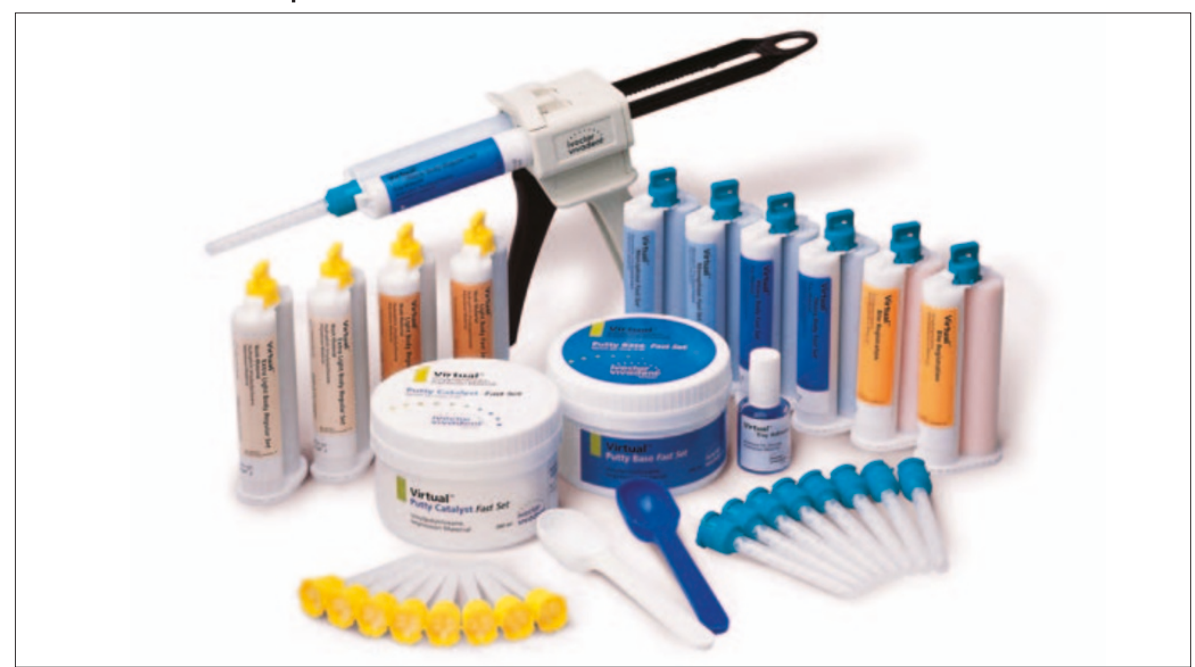

Ivoclar Vivadent's Virtual is a vinylpolysiloxane impression material which is available in fast or normal working and setting times, and in a range of consistencies.

It allows the user to take impressions using various techniques as required by different cases. Virtual is a moisture toler- ant putty and wash system. Its thixotropic nature allows the material to flow under pressure and hold its shape when not under pressure.

The product comes in $300 \mathrm{ml}$ base and catalyst tubs with the wash coming in $50 \mathrm{ml}$ cartridges.

Reader response number 60

\section{Finance plan for children}

Practice Plan can help practices to offer children's plans designed to improve the standard of oral health and dental care in young patients, it claims.

The plan can encompass regular hygiene and dental appointments, accompanied by the appropriate advice on care for gums and teeth, and regular radiographs if required.

There is flexibility to

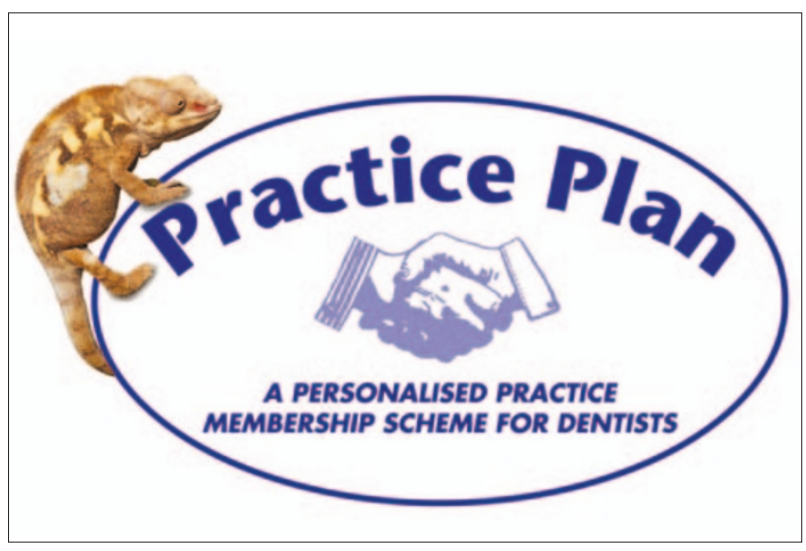
include visits to an oral health educator or to include fissure sealants and also to adapt the nature of the visits for various age ranges.

All options incorporate worldwide trauma and emergency call-out cover for children and Practice Plan can help with the design of a letter communicating the benefits of such a scheme to parents.

Reader response number 61

\section{Single pour system}

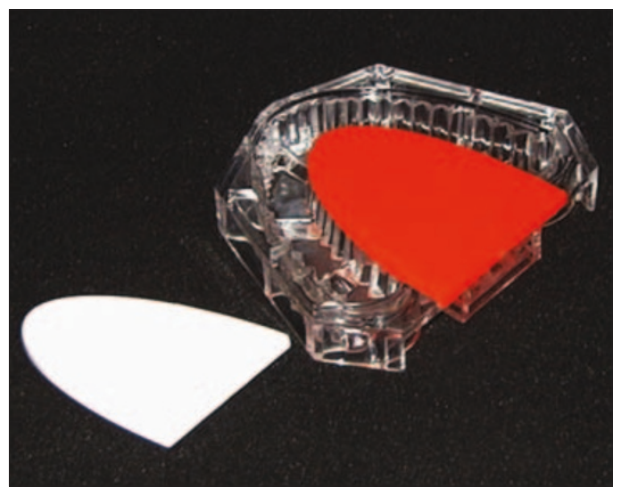

The Krystal Model Tray System offers a simple, single pour system and is quick to prepare without the need for rebasing, gluing or pinning, its manufacturers assert.

The tray produces accurate models with positive retention of die segments, has a central tongue model retainer with simple, positive locks for all dies, a unique casting tongue, a snap free tongue removal tool, and a removable tray base. Reader response number 62 